\title{
BMJ Open Effect of regulating gut microbiota using probiotics on functional changes in the brain: protocol for a systematic review
}

\author{
Lu Liu (D) , ${ }^{1}$ Xixiu Ni, ${ }^{1}$ Tian Tian, ${ }^{1}$ Xiao Li, ${ }^{1}$ Fengmei Li, ${ }^{1}$ Mingsheng Sun, ${ }^{1}$ \\ Jiao Chen (D) , ${ }^{1}$ SiYuan Zhou, ${ }^{1,2}$ Ling Zhao (D) ${ }^{1,2}$
}

To cite: Liu L, Ni X, Tian T, et al. Effect of regulating gut microbiota using probiotics on functional changes in the brain: protocol for a systematic review. BMJ Open 2020;10:e037582. doi:10.1136/ bmjopen-2020-037582

- Prepublication history and additional material for this paper are available online. To view these files, please visit the journal online (http://dx.doi. org/10.1136/bmjopen-2020037582).

SZ and LZ contributed equally.

Received 08 February 2020

Revised 03 April 2020

Accepted 23 June 2020

Check for updates

(c) Author(s) (or their employer(s)) 2020. Re-use permitted under CC BY-NC. No commercial re-use. See rights and permissions. Published by BMJ.

${ }^{1}$ Acupuncture and Tuina School, Chengdu University of Traditional Chinese Medicine, Chengdu, China

${ }^{2}$ Acupuncture \& Chronobiology Key Laboratory of Sichuan Province, Chengdu, China

Correspondence to

Professor Ling Zhao;

zhaoling@cdutcm.edu.cn and

Dr. SiYuan Zhou;

zsy@cdutcm.edu.cn

\section{ABSTRACT}

Introduction There is a growing number of randomised controlled trials (RCTs) that focus on functional changes in the brain detected by functional MRI (fMRI) and gut microbiota composition changes after using probiotics. However, the effect of probiotics on functional changes in the brain through gut microbiota remains controversial in existing RCTs. Furthermore, to our knowledge, there is no systematic review to evaluate the effect of probiotics on functional changes in the brain through gut microbiota. Therefore, we aim to summarise literatures evaluating the potential association between probiotics, gut microbiota and functional changes in the brain to elucidate whether probiotics influence gut microbiota and affect functional changes in the brain through gut microbiota.

Methods and analysis China National Knowledge Infrastructure, Wanfang Data, VIP Databases (the Chongqing VIP Chinese Science and Technology Periodical Database), SinoMed, PubMed, Web of Science, MEDLINE (The National Library of Medicine), EMBASE (Excerpt Medica Database), Scopus, the Cochrane Central Register of Controlled Trials and ClinicalTrials.gov will be searched until July 2019. The Grey Literature in Europe (OpenSIGLE) database and Google search engine will also be used. The reference lists of each included study will be reviewed to determine whether there are any further relevant studies. RCTs using probiotics compared with a placebo/control will be included. We will use risk of bias assessment and the Grading of Recommendations Assessment, Development and Evaluation (GRADE) system to assess the quality of evidence. The results of the systematic review will be synthesised narratively in the domains of the three primary outcome measures: (1) Increased/decreased activity in brain regions or altered functional connectivity (FC) of brain detected by $\mathrm{fMRI}$ and their association with changes in behaviour, gastrointestinal/emotional symptoms after using probiotics. (2) Changes in composition and diversity of the gut microbiota and their association with changes in behaviour, gastrointestinal/emotional symptoms after using probiotics. (3) Increased/decreased activity in brain regions or altered FC of brain detected by FMRI and the changes in composition or diversity of the gut microbiota after administration of probiotics.

Ethics and dissemination The results will be disseminated through a peer-reviewed publication. As no private and confidential patient data will be included in the
Strengths and limitations of this study

This is the first systematic review assessing the potential association between probiotics, gut microbiota and functional changes in the brain.

- The study design adheres to all relevant guidelines for systematic reviews and meta-analyses.

- The quality of evidence will be assessed by the Grading of Recommendations Assessment, Development and Evaluation system.

- This systematic review will have inherent limitations related to the included studies such as risk of bias, methodological inconsistencies and incomplete outcome data.

- We will only retrieve data from Chinese and English databases, which may limit available data or result in language bias.

reporting, there are no ethical considerations associated with this protocol.

PROSPERO registration number CRD42019145114.

\section{BACKGROUND}

Probiotics are defined as 'live microorganisms' that provide health benefits to the host when consumed in adequate amounts. ${ }^{1}$ The use of probiotics has emerged as a principal approach to maintain the balance of the human gut microbiota. ${ }^{2}$ Probiotics have a strong ability to modulate the gut microbiota composition in healthy subjects, ${ }^{3}{ }^{4}$ leading to a significant reduction in several bacterial genera directly involved in the onset of gastrointestinal diseases. ${ }^{3}$ Furthermore, probiotics regulate the gut microbiota of patients with gastrointestinal diseases and improve clinical symptoms. ${ }^{56}$ These findings show that probiotics can be used to treat gastrointestinal diseases by maintaining or changing the gut microbiota.

The gut microbiota includes bacteria, fungi, archaea, protozoa and viruses that 
interact with the host and each other to affect the host's physiology and health. ${ }^{7}$ The microbiota has recently emerged as a key player in the gut-brain axis. This interaction between the brain and the microbiota in both animals and humans has led to the recognition of a new term called 'microbiota-gut-brain axis'. ${ }^{8}$ This interaction is bidirectional, meaning that disturbance in the complex community of microbiota (dysbiosis) can affect the brain and vice versa. ${ }^{9} \mathrm{~A}$ meta-analysis that was performed on 13 studies up until 2016 has shown that there were significant differences in the levels of Lactobacillus, Bifidobacterium and Faecalibacterium prausnitzii in patients with irritable bowel syndrome (IBS) compared with healthy controls. ${ }^{10}$ Alteration in gut microbiota may contribute to pathogenesis of patients with IBS by altering the gut-brain axis. ${ }^{11}$ Patients with neurological disorders were linked to gastrointestinal dysfunction and changes in gut microbiota. ${ }^{12}$ And in another clinical trial, Candida albicans exposure has been found to be associated with gastrointestinal dysfunction and impaired cognitive ability. ${ }^{13}$ The underlying signalling mechanisms of these communicating networks between the gut flora and the gut-brain axis have been of special interest to researchers who are seeking potential therapeutic interventions. ${ }^{9}$

The ingestion of probiotics by healthy humans showed diminished psychological symptoms, including anxiety symptoms, ${ }^{14}$ while probiotic intervention significantly ameliorated the severity of depression in patients with major depressive disorder ${ }^{15}$ and patients with IBS. $^{16}$ The above results show that gut microbiota are related to various diseases, and that modulating gut microbiota through probiotics may offer a potential treatment option. However, most of the psychological assessments in clinical trials ${ }^{141517}$ were based solely on self-reported measures, hence firm conclusions regarding the effects of probiotics cannot be drawn from these findings.

Given the difficulty of studying the brain at the cellular level in humans, neuroimaging has emerged as a tool for increasing our understanding of the brain. Advances in computational biology are beginning to explain how these multifaceted and complex systems interact with each other. ${ }^{1819}$ Neuroimaging tools as outcome measures are more objective than self-reported measures, which is an advantage over self-reported measures. Functional MRI (fMRI) is able to measure moment-to-moment alterations in the blood oxygen content (the blood oxygen level dependent signal) ${ }^{20}$ Furthermore, it enables us to identify real time changes in neurological activity and correlate these with changes in behaviour or perception. ${ }^{21-23}$ fMRI has been used successfully to identify differences in brain function in gastrointestinal disease states, such as IBS and inflammatory bowel disease, as well as in healthy people before and after chronic ingestion of probiotics. ${ }^{20} 24$ fMRI is effective predominantly because of its non-invasiveness, and it can accurately and intuitively observe the location and range of brain function activity from the whole level under physiological conditions. ${ }^{26}$ Using task-based MRI or resting-state
fMRI (rs-fMRI), enables us to examine whether probiotics influence the gut microbiota and affect activity in brain regions or functional connectivity (FC) of brain through the gut microbiota. It helps us better understand the microbiota-gut-brain axis, to potentially help develop therapeutic methods for central nervous system diseases in the future.

Recently, there were randomised controlled trials (RCTs) that focused on increased/decreased activity in brain regions ${ }^{24} 2728$ or altered FC of brain ${ }^{24} 29$ detected by task-based fMRI or rs-fMRI after using probiotics in healthy subjects. However, some studies have found changes in gut microbiota, ${ }^{27} 28$ behaviour, gastrointestinal/emotional symptoms, ${ }^{27}$ while others have not. ${ }^{24}$ Furthermore, to our knowledge, there is no systematic review to evaluate the effect of probiotics on functional changes in the brain through gut microbiota. Therefore, we plan to systematically analyse all published RCTs in the medical literature to assess the effects of probiotics on the gut microbiota and functional changes in the brain. This will improve our understanding of the microbiotagut-brain axis. Moreover, a deeper understanding of the underlying mechanisms will help refine the clinical use of probiotic supplements in the future.

\section{OBJECTIVES}

The objective of this systematic review is to summarise and evaluate the literature evaluating the potential association between probiotics, gut microbiota and increased/ decreased activity in brain regions or altered FC of brain in healthy subjects after taking probiotics of any type, dosage, time point and duration. To this end, we aim to:

1. Evaluate the relationship between the changes in behaviour, gastrointestinal/emotional symptoms and increased/decreased activity in brain regions or altered FC of brain after probiotics' administration.

2. Evaluate the relationship between the behaviour, gastrointestinal/emotional symptoms and the changes in composition or diversity of gut microbiota after probiotics' administration.

3. Evaluate the relationship between increased/decreased activity in brain regions or altered FC of brain and the changes in composition or diversity of gut microbiota after probiotics' administration.

We aim to determine whether probiotics influence gut microbiota and affect functional changes in the brain through the gut microbiota.

\section{METHODS}

\section{Retrieval strategy}

The following databases will be searched until July 2019 for relevant RCTs: China National Knowledge Infrastructure (CNKI), Wanfang Data, VIP Databases, SinoMed, PubMed, Web of Science, MEDLINE, EMBASE, Scopus, the Cochrane Central Register of Controlled Trials and ClinicalTrials.gov. The Grey Literature in Europe 
(OpenSIGLE) database and Google search engine will also be searched. Furthermore, reference lists of each included study will be reviewed to determine whether there are any further relevant studies. The search terms will include probiotics (eg, 'probiotics' or 'culturelle' or 'bifidobacteria' or 'lactobailli' or 'escherichia coli' or 'yoghurt' or 'yeast' or 'saccharomyces' or 'bacillus' or 'enterococcus' or 'streptococcus' or 'fermented milk' or 'sour milk ' or 'gastrointestinal microbiome' or 'gut microbiota' or 'gut microbiome' or 'gut microflora' or 'intestinal microflora' or 'intestinal bacteria' or 'intestinal microbiome' or 'microbiome' or 'microbiota' or 'bacteria' or 'flora'), fMRI (eg, 'MRI' or 'fMRI' or 'functional MRI' or 'neuroimaging' or 'MRI' or 'brain activity' or 'MR tomography' or 'NMR Imaging' or 'MR' or 'independent component analysis' or ' functional network connectivity' or 'amplitude of low frequency fluctuations' or 'regional homogeneity' or 'functional connectivity' or 'central' or 'brain' or 'neuro' or 'ALFF' or 'FALFF' or 'REHO' or 'ROI' or 'FC' or 'ICA'). The following terms will be used in the Chinese database searches: 'Yi sheng jun', 'Chang dao jun qun', 'Jun qun', 'Nao gong neng cheng xiang', 'Gong neng xing ci gong zhen', 'He ci gong zhen cheng xiang' and 'He ci'. The search strategy for the PubMed database is shown in online supplementary table 1 ; this strategy will be modified appropriately for other databases.

\section{Inclusion criteria}

1. Type of study: randomised clinical controlled trials.

2. Participants: healthy individuals.

3. Interventions: probiotics compared with a placebo/ control.

4. Outcome measures: the primary outcome measures for this review will be the assessment of functional changes in the brain, which were measured by fMRI, as they relate to gut microbiota regulated by probiotics. Three different primary outcome measures will be obtained from the following primary research: (1) Increased/decreased activity in brain regions or altered FC of brain detected by fMRI and their association with changes in behaviour, gastrointestinal/emotional symptoms after using probiotics. (2) Changes in composition and diversity of the gut microbiota and their association with changes in behaviour, gastrointestinal/emotional symptoms after using probiotics. (3) Increased/decreased activity in brain regions or altered FC of brain detected by fMRI and the changes in composition or diversity of the gut microbiota after administration of probiotics. Secondary outcomes: adverse events and participants' compliance during the trial.

\section{Exclusion criteria}

1. Non-randomised clinical controlled trials.

2. Editorials, literature reviews and meta-analyses.

3. Duplicate publications.

4. Studies without full report of study results or primary data.

\section{Study selection}

All the studies retrieved through the search strategy will first be screened based on title and abstract using ENDNOTE (ENDNOTE is a reference management software that helps a lot in collecting or curating research materials, and formatting bibliographies.) which can remove duplicates. Two reviewers will independently screen all titles and abstracts according to the inclusion criteria. Any disagreements will be resolved by discussion or by a third reviewer. Full texts of potentially eligible studies based on title and abstract will be screened for eligibility by two reviewers independently; disagreements will be resolved by discussion or by a third reviewer if necessary. Reasons for study exclusions will be recorded.

\section{Data extraction}

Data will be extracted from each eligible study including the following information: authors; year of publication; journal; sample size; demographic characteristics of sample (age, ethnicity, sex); any information (eg, type, duration, dose, time point) we can get on the intervention group and control/placebo group; data analysis strategy used; fMRI data analysis strategy used; outcomes and conclusions.

\section{Quality assessment}

Risk of bias assessment: the included studies will be assessed by two independent investigators using the Cochrane Risk of Bias Assessment Tool. ${ }^{30}$ The assessment will include contents of random sequence generation, allocation concealment, blinding (of participants, personnel and outcome assessors), incomplete outcome data and selective outcome reporting and other sources of bias. The investigators' judgement will be categorised as 'Low risk', 'High risk' or 'Unclear risk' of bias. The disagreements will be resolved by discussion or a by third analyser if necessary.

Quality of evidence assessment: two independent reviewers will assess overall quality of evidence using the Grading of Recommendations Assessment,Development and Evaluation (GRADE) system, examining the quality of the literature in five domains-risk of bias, publication bias, consistency, directness and precision. ${ }^{31}$ This system has four levels of classification ('high', 'moderate', 'low' and 'very low') to which each study can be assigned based on different factors. Disagreements will be resolved by discussion or by a third analyser if necessary.

\section{Strategy of data synthesis}

The results of the systematic review will be synthesised narratively in the domains of the three primary outcome measures: (1) Increased/decreased activity in brain regions or altered FC of brain and their association with changes in behaviour, gastrointestinal/ emotional symptoms after using probiotics. (2) Changes in composition and diversity of the gut microbiota and their association with changes in behaviour, gastrointestinal/emotional symptoms after using probiotics. (3) 
Increased/decreased activity in brain regions or altered FC of brain and the changes in composition or diversity of the gut microbiota after administration of probiotics and one secondary outcome measure: the relevant adverse events and participants' compliance. We will extract the increased/decreased activity in brain regions or altered FC of brain to evaluate whether probiotics are effective in activating/deactivating particular brain regions or are effective in altering FC of brain, where the functional changes in the brain are. Additionally, we will evaluate the relationship between the changes in behaviour, gastrointestinal/emotional symptoms and the increased/decreased activity in brain regions or altered FC of brain after administration of probiotics. If possible from the number of studies available, we will use narrative review to explicate whether a 'dose-dependent' or 'typedependent' relationship can be identified between probiotic supplements and functional changes in the brain. We will also evaluate the relationship between the changes in behaviour, gastrointestinal/emotional symptoms and the changes in composition or diversity of the gut microbiota after administration of probiotics, and evaluate the relationship between the increased/decreased activity in brain regions or altered $\mathrm{FC}$ of brain and the changes in composition or diversity of the gut microbiota after administration of probiotics. Finally, we will record all the adverse events and participants' compliance during the trial to assess the safety and popularisation of probiotic supplements, which can be regarded as a new potential therapeutic method for central nervous system diseases in the future. To fully assess for gaps in evidence, we will do the risk of bias assessment, and the strength of evidence for this conclusion will be determined by the items evaluated in the GRADE framework described above. We will highlight areas that need further study and give suggestions for future studies.

\section{Registration and reporting of results}

We registered this study in the PROSPERO international prospective register of systematic reviews (PROSPERO is an international database of prospectively registered systematic reviews in health and social care, welfare, public health, education, crime, justice, and international development, where there is a health related outcome.) in July 2019. ${ }^{32}$ This protocol was drafted according to the Preferred Reporting Items for Systematic Reviews and Meta-Analyses Protocols (PRISMA-P).$^{33}$ The methods and results of the systematic review will be reported in accordance with the PRISMA-P guidelines. ${ }^{34}$

\section{Patient and public involvement}

No patients or public will be involved in this systematic review protocol.

\section{DISCUSSION}

According to the Global Burden of Disease Study, 322 million and 264 million people worldwide suffered from depression and anxiety, respectively in 2015. This is an increase of $18.4 \%$ and $14.9 \%$ over the 2005 figures. ${ }^{35}$ There were more than 80 million stroke survivors in the world, ${ }^{36} 43.8$ million people with dementia, ${ }^{37} 45.9$ million patients with an active epilepsy, ${ }^{38}$ and 6.1 million individuals with Parkinson's disease. ${ }^{39}$ Globally, in 2016, neurological disorders were the leading cause of disability (276 million disability-adjusted life years) and the second leading cause of deaths (9 million) in the world. ${ }^{40}$ With the increasing annual growth in industrial production of food containing probiotics worldwide, the interest in elucidating how changes in the gut microbiota and functional changes in brain as a result of ingestion of probiotics can serve as a new way for treating subjects with central nervous system diseases is increasing. In our systematic review, we will synthesise the evidence evaluating the association between probiotic supplements and functional changes in the brain and gut microbiota changes in humans to decipher whether probiotics influence functional changes in the brain and whether the effect of probiotics on functional changes in the brain is associated with changes in gut microbiota. This review provides a new way of thinking about the treatment of central nervous system diseases and health maintenance. To our knowledge, this will be the first systematic review focused on this topic.

Additionally, from the adverse events and participants' compliance with probiotic supplements, we can accumulate evidence for the popularisation of probiotic therapy, which may be used to treat central system diseases in the future. This can help better understand the clinical application, effectiveness and safety of probiotics.

Furthermore, we will determine gaps or uncertainties in the existing literature, which require further study. However, given the potential implications of such an association, this review will provide a solid foundation for the design and implementation of future studies that can better clarify the relationship between probiotics and functional changes in the brain and gut microbiota and in humans.

Acknowledgements The authors thank Michal Bell, from Liwen Bianji, Edanz Editing China (www. liwenbianji. cn/ ac) for editing the English text of a draft of this manuscript.

Contributors LL developed the search strategy, $T T$ and XL will search the databases and screen the eligibility of the retrieved studies. FL and XN will extract information from the eligible studies and prepare the information for data analysis. JC, MS and SZ will perform the data analysis. LL and LZ wrote the first draft of the protocol. In practice, LZ will monitor each procedure of the review and is responsible for quality control. All authors read the article and approved it for publication. All authors read and approved the final manuscript and order of authorship.

Funding The review is supported by the National Key Research and Development Project (Grant No.2019YFC1709701), the National Natural Science Foundation of China (Grant No.81722050, 81973962) and the Interdisciplinary Program of Chengdu University of Traditional Chinese Medicine (Grant No.CZYJC1901). Funders and sponsors have no role in the design of this protocol.

Competing interests None declared.

Patient and public involvement Patients and/or the public were not involved in the design, or conduct, or reporting, or dissemination plans of this research. 
Patient consent for publication Not required.

Provenance and peer review Not commissioned; externally peer reviewed.

Open access This is an open access article distributed in accordance with the Creative Commons Attribution Non Commercial (CC BY-NC 4.0) license, which permits others to distribute, remix, adapt, build upon this work non-commercially, and license their derivative works on different terms, provided the original work is properly cited, appropriate credit is given, any changes made indicated, and the use is non-commercial. See: http://creativecommons.org/licenses/by-nc/4.0/.

\section{ORCID iDs}

Lu Liu http://orcid.org/0000-0003-2196-7627

Jiao Chen http://orcid.org/0000-0003-0830-6242

Ling Zhao http://orcid.org/0000-0002-3955-0072

\section{REFERENCES}

1 Hill C, Guarner F, Reid G, et al. Expert consensus document. The International scientific association for probiotics and prebiotics consensus statement on the scope and appropriate use of the term probiotic. Nat Rev Gastroenterol Hepatol 2014;11:506-14.

2 Hardy H, Harris J, Lyon E, et al. Probiotics, prebiotics and immunomodulation of gut mucosal defences: homeostasis and immunopathology. Nutrients 2013;5:1869-912.

3 Toscano M, De Grandi R, Miniello VL, et al. Ability of Lactobacillus kefiri LKF01 (DSM32079) to colonize the intestinal environment and modify the gut microbiota composition of healthy individuals. Dig Liver Dis 2017;49:261-7.

4 Toscano M, De Grandi R, Stronati L, et al. Effect of Lactobacillus rhamnosus HN001 and Bifidobacterium longum BB536 on the healthy gut microbiota composition at phyla and species level: A preliminary study. World J Gastroenterol 2017;23:2696-704.

5 Chen L, Xu W, Lee A, et al. The impact of Helicobacter pylori infection, eradication therapy and probiotic supplementation on gut microenvironment homeostasis: an open-label, randomized clinical trial. EBioMedicine 2018;35:87-96

6 Mezzasalma V, Manfrini E, Ferri E, et al. A randomized, doubleblind, placebo-controlled trial: the efficacy of multispecies probiotic supplementation in alleviating symptoms of irritable bowel syndrome associated with constipation. Biomed Res Int 2016;2016:1-10.

7 Claesson MJ, Jeffery IB, Conde S, et al. Gut microbiota composition correlates with diet and health in the elderly. Nature 2012;488:178-84.

8 Mayer EA. Gut feelings: the emerging biology of gut-brain communication. Nat Rev Neurosci 2011;12:453-66.

9 Mukhtar K, Nawaz H, Abid S. Functional gastrointestinal disorders and gut-brain axis: what does the future hold? World J Gastroenterol 2019;25:552-66.

10 Liu H-N, Wu H, Chen Y-Z, et al. Altered molecular signature of intestinal microbiota in irritable bowel syndrome patients compared with healthy controls: a systematic review and meta-analysis. Dig Liver Dis 2017:49:331-7.

11 Chong PP, Chin VK, Looi CY, et al. The Microbiome and Irritable Bowel Syndrome - A Review on the Pathophysiology, Current Research and Future Therapy. Front Microbiol 2019;10:1136.

12 Strati F, Cavalieri D, Albanese D, et al. Altered gut microbiota in Rett syndrome. Microbiome 2016;4:41.

13 Severance EG, Gressitt KL, Stallings CR, et al. Candida albicans exposures, sex specificity and cognitive deficits in schizophrenia and bipolar disorder. NPJ Schizophr 2016;2:16018.

14 Messaoudi M, Lalonde R, Violle N, et al. Assessment of psychotropic-like properties of a probiotic formulation (Lactobacillus helveticus R0052 and Bifidobacterium longum R0175) in rats and human subjects. Br J Nutr 2011;105:755-64.

15 Akkasheh G, Kashani-Poor Z, Tajabadi-Ebrahimi M, et al. Clinical and metabolic response to probiotic administration in patients with major depressive disorder: a randomized, double-blind, placebo-controlled trial. Nutrition 2016;32:315-20.

16 Pinto-Sanchez MI, Hall GB, Ghajar K, et al. Probiotic Bifidobacterium longum NCC3001 reduces depression scores and alters brain activity: a pilot study in patients with irritable bowel syndrome. Gastroenterology 2017;153:448-59.

17 Ostadmohammadi V, Jamilian M, Bahmani F, et al. Vitamin D and probiotic co-supplementation affects mental health, hormonal, inflammatory and oxidative stress parameters in women with polycystic ovary syndrome. J Ovarian Res 2019;12:5.

18 Malan-Muller S, Valles-Colomer M, Raes J, et al. The gut microbiome and mental health: implications for anxiety- and trauma-related disorders. OMICS 2018;22:90-107.

19 Cong X, Henderson WA, Graf J, et al. Early life experience and gut microbiome: the Brain-Gut-Microbiota signaling system. Adv Neonatal Care 2015;15:314-23. quiz E1-2.

20 Tillisch K, Mayer EA, Labus JS. Quantitative meta-analysis identifies brain regions activated during rectal distension in irritable bowel syndrome. Gastroenterology 2011;140:91-100.

21 Wang $\mathrm{H}$, Lee I-S, Braun C, et al. Effect of probiotics on central nervous system functions in animals and humans: a systematic review. J Neurogastroenterol Motil 2016;22:589-605.

22 Lener MS, Niciu MJ, Ballard ED, et al. Glutamate and gammaaminobutyric acid systems in the pathophysiology of major depression and antidepressant response to ketamine. Biol Psychiatry 2017;81:886-97.

23 Al Omran Y, Aziz Q. Functional brain imaging in gastroenterology: to new beginnings. Nat Rev Gastroenterol Hepatol 2014;11:565-76.

24 Tillisch K, Labus J, Kilpatrick L, et al. Consumption of fermented milk product with probiotic modulates brain activity. Gastroenterology 2013;144:1394-401. 1401.e1-4

25 Agostini A, Filippini N, Cevolani D, et al. Brain functional changes in patients with ulcerative colitis: a functional magnetic resonance imaging study on emotional processing. Inflamm Bowel Dis 2011;17:1769-77.

26 Smitha KA, Akhil Raja K, Arun KM, et al. Resting state fMRI: a review on methods in resting state connectivity analysis and resting state networks. Neuroradiol J 2017;30:305-17.

27 Bagga D, Reichert JL, Koschutnig K, et al. Probiotics drive gut microbiome triggering emotional brain signatures. Gut Microbes 2018;9:1-11.

28 Tillisch K, Mayer EA, Gupta A, et al. Brain structure and response to emotional stimuli as related to gut microbial profiles in healthy women. Psychosom Med 2017;79:905-13.

29 Bagga D, Aigner CS, Reichert JL, et al. Influence of 4-week multistrain probiotic administration on resting-state functional connectivity in healthy volunteers. Eur J Nutr 2019;58:1821-7.

30 Higgins JPT, Altman DG, Gøtzsche PC, et al. The Cochrane collaboration's tool for assessing risk of bias in randomised trials. BMJ 2011;343:d5928.

31 Guyatt GH, Oxman AD, Schünemann HJ, et al. GRADE guidelines: a new series of articles in the Journal of clinical epidemiology. J Clin Epidemiol 2011;64:380-2.

32 Liu L, Xi Xiu NI, TIAN T, et al. Effect of regulating gut microbiota using probiotics on brain activity: protocol of a systematic review. PROSPERO, 2019. Available: http://www.crd.york.ac.uk/ PROSPERO/display_record.asp?ID=CRD42019145114

33 Shamseer L, Moher D, Clarke M, et al. Preferred reporting items for systematic review and meta-analysis protocols (PRISMA-P) 2015 elaboration and explanation. BMJ 2015;350:g7647.

34 Moher D, Shamseer L, Clarke M, et al. Preferred reporting items for systematic review and meta-analysis protocols (PRISMA-P) 2015 statement. Syst Rev 2015;4:1.

35 Vos T, Allen C, Arora M, et al. Global, regional, and national incidence, prevalence, and years lived with disability for 310 diseases and injuries, 1990-2015: a systematic analysis for the global burden of disease study 2015. The Lancet 2016;388:1545-602.

36 GBD 2016 Stroke Collaborators. Global, regional, and national burden of stroke, 1990-2016: a systematic analysis for the global burden of disease study 2016. Lancet Neurol 2019;18:439-58.

37 GBD 2016 Dementia Collaborators. Global, regional, and national burden of Alzheimer's disease and other dementias, 1990-2016: a systematic analysis for the global burden of disease study 2016. Lancet Neurol 2019;18:88-106.

38 GBD 2016 Epilepsy Collaborators. Global, regional, and national burden of epilepsy, 1990-2016: a systematic analysis for the global burden of disease study 2016. Lancet Neurol 2019;18:357-75.

39 GBD 2016 Parkinson's Disease Collaborators. Global, regional, and national burden of Parkinson's disease, 1990-2016: a systematic analysis for the global burden of disease study 2016. Lancet Neurol 2018;17:939-53.

40 GBD 2016 Neurology Collaborators. Global, regional, and national burden of neurological disorders, 1990-2016: a systematic analysis for the global burden of disease study 2016. Lancet Neurol 2019;18:459-80. 